\title{
Performance characterization of the HiCIAO instrument for the Subaru Telescope
}

Ryuji Suzuki $^{a b}$, Tomoyuki Kudo ${ }^{b c}$, Jun Hashimoto ${ }^{d}$, Joseph Carson ${ }^{e}$, Sebastian Egner $^{b}$, Miwa Goto $^{e}$, Masayuki Hattori ${ }^{b}$, Yutaka Hayano ${ }^{b}$, Klaus Hodapp $f$, Meguru Ito ${ }^{b}$, Masanori Iye $^{c}$, Shane Jacobson ${ }^{f}$, Ryo Kandori ${ }^{c}$, Nobuhiko Kusakabe ${ }^{c}$, Masayuki Kuzuhara ${ }^{g}$, Taro Matsuo ${ }^{c}$, Michael Mcelwain ${ }^{h}$, Jun-ichi Morino ${ }^{c}$, Shin $\mathrm{Oya}^{b}$, Yoshihiko Saito ${ }^{b}$, Richard Shelton ${ }^{f}$, Vern Stahlberger $^{f}$, Hiroshi Suto $^{c}$, Hideki Takami $^{b}$, Christian Thalmann $^{e}$, Makoto Watanabe $^{i b}$, Hubert Yamada ${ }^{f}$, and Motohide Tamura ${ }^{c}$

${ }^{a}$ Thirty Meter Telescope Observatory Corporation, 2632 E.Washington Blvd., Pasadena, CA, U.S.A 91107;

${ }^{b}$ Subaru Telescope, 650 North A'ohoku Place, Hilo, HI, U.S.A 96720;

${ }^{c}$ National Astronomical Observatory of Japan, 2-21-1 Osawa, Mitaka, Tokyo, Japan 181-8588;

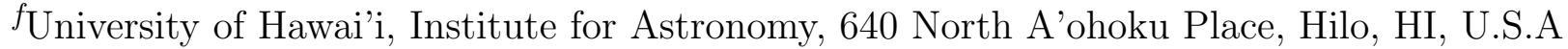
96720;

${ }^{e}$ Max Planck Institute for Astronomy, Konigstuhl 17, Heidelberg, Germany D-69117;

${ }^{d}$ Graduate University for Advanced Studies, 2-21-1 Osawa, Mitaka, Tokyo, Japan 181-8588;

${ }^{g}$ The University of Tokyo, 2-21-1 Osawa, Mitaka, Tokyo, Japan 181-8588;

${ }^{h}$ Princeton University, Department of Astrophysical Sciences, Peyton Hall, Princeton, NJ, U.S.A $08544-1001$;

${ }^{i}$ Hokkaido University, Department of Cosmoscience, Kita-10-Nishi-8, Kita, Sapporo, Hokkaido, Japan 060-0811

\begin{abstract}
HiCIAO is a high contrast instrument which works with a 188 element adaptive optics system (AO188) for the Subaru Telescope. The instrument is specifically designed for extrasolar planet search and studies of protoplanetary or debris disks. A coronagraph technique and some differential observing modes implemented in HiCIAO extract faint objects from the sea of speckle around bright stars. We describe the instrument performances verified in the laboratory and during the commissioning period. Readout noise with a correlated double sampling method is $15 \mathrm{e}^{-}$using the Sidecar ASIC controller with the HAWAII-2RG detector array. The readout noise becomes as low as $5 \mathrm{e}^{-}$with a multiple sampling method. Strehl ratio obtained by AO188+HiCIAO on the sky is 0.4 and 0.7 in the $H$ and $K$-band, respectively, with $R \sim 5$ stars under median seeing conditions. Image distortion is correctable to 7 milli-arcsec level using the ACS data as a reference image. Some examples of contrast performances in all observing modes are presented from a limited number of observations. An ADI observation for HR 8799 shows a clear detection of three known planets, demonstrating high contrast capability of AO188+HiCIAO.
\end{abstract}

Keywords: near-infrared, camera, coronagraph, high contrast, adaptive optics

\section{INTRODUCTION}

National Astronomical Observatory of Japan has developed a new high contrast instrument for the $8.2 \mathrm{~m}$ Subaru Telescope under collaboration with the University of Hawaii, Institute for Astronomy. The instrument called HiCIAO is designed to work with a newly developed 188 element adaptive optics system (AO188; Hayano et

Further author information: (Send correspondence to R.S.)

R.S.: E-mail: ryuji@tmt.org, Telephone: 16263958956 


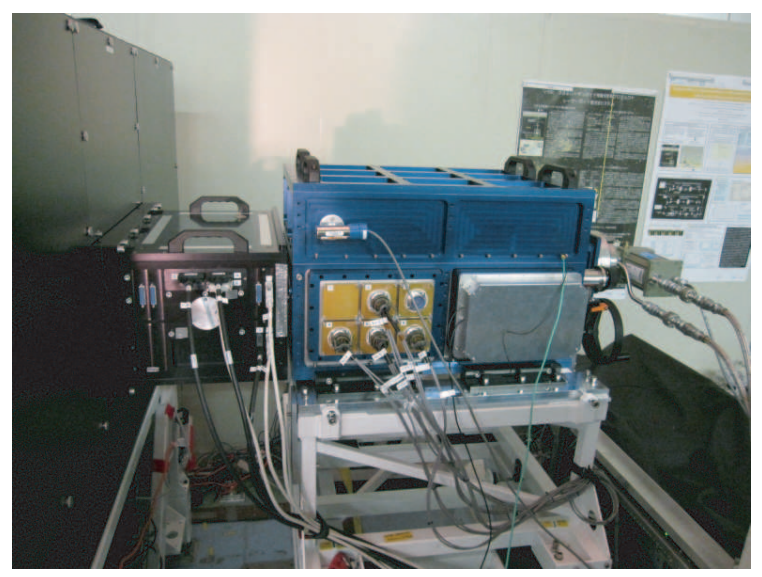

Figure 1. HiCIAO at the observing position on the Nasmyth floor of the Subaru Telescope. A black wall on the left side is $\mathrm{AO} 188$.

al. 2010 ${ }^{1}$ ), and is specific to extrasolar planet search and detailed disk sciences. The basic concept of the instrument is utilizing a high quality wavefront from the adaptive optics, coronagraph, and some differential imaging techniques to enhance the contrast in the vicinity of bright stars where speckle noise hinders the planet or disk detection. The instrument is an infrared imager which consists of a warm coronagraph/collimator module and a cryogenic infrared camera module (Figure 1). The fundamental instrument parameters are summarized as $20^{\prime \prime} \times 20^{\prime \prime}$ field of view with 10 milli-arcsec pixel scale and wavelength coverage from $0.80 \mu \mathrm{m}$ to $2.4 \mu \mathrm{m}$. A simple Lyot type coronagraph with hard-edged opaque circular occulting masks and an undersized Lyot stop are implemented in the warm coronagraph module. The warm coronagraph module also includes single and double Wollaston prisms which split the light into two or four directions for the differential imaging techniques. The cryogenic infrared camera module houses filters and a $2048 \times 2048$ pixel HAWAII-2RG infrared detector array (Teledyne Scientific \& Imaging LLC.). Two filter wheels are located near the pupil and the detector for a spectral differential imaging mode as well as a normal imaging mode. Detail of the instrument concept and design is described in Hodapp et al. $\left(2006 ;^{2} 2008^{3}\right)$ and Tamura et al. (2006). ${ }^{4}$

HiCIAO currently supports a normal imaging mode (DI mode) and three differential imaging modes with different techniques: Polarimetric Differential Imaging mode (PDI mode), Spectral Differential Imaging mode (SDI mode), and Angular Differential Imaging mode (ADI mode). The ADI mode can cooperate with any of other observing modes such as DI+ADI, PDI+ADI, and SDI+ADI mode. An example of the images in each observing mode is shown in Figure 2. The DI mode is a normal imaging mode with $20^{\prime \prime} \times 20^{\prime \prime}$ field of view with a set of standard broadband filters in the near-infrared regime. In the PDI mode, two images with $10^{\prime \prime} \times 20^{\prime \prime}$ each corresponding to two directions of polarization are obtained at the same time using the single Wollaston
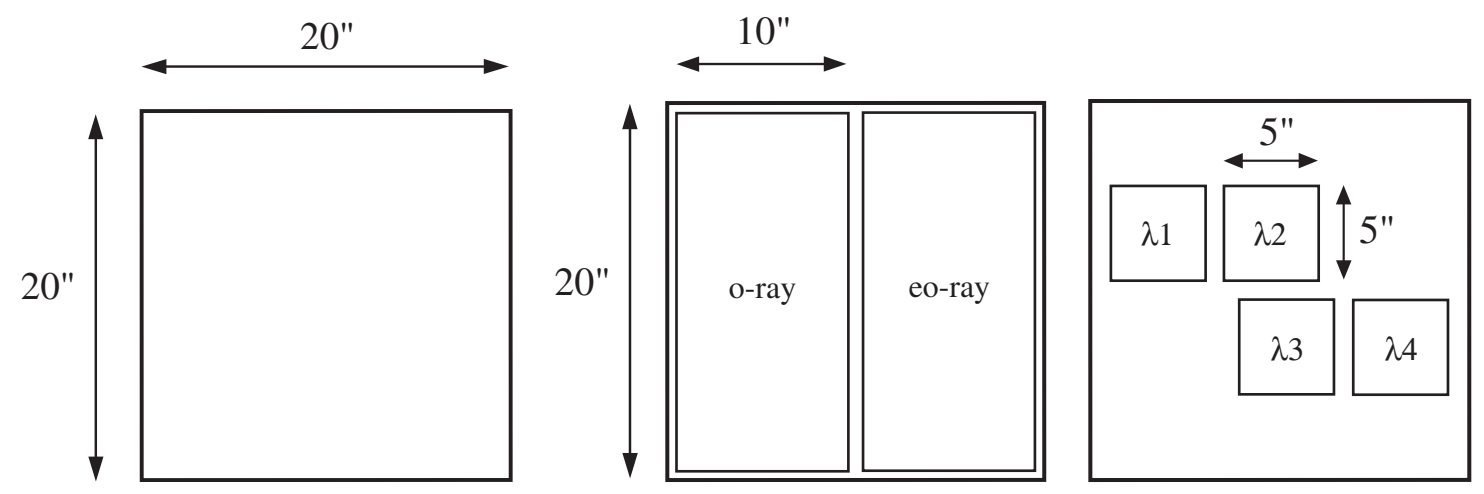

Figure 2. Arrangement of the image in the DI mode (left), PDI mode (center), and SDI mode (right). 
prism. The single Wollaston prism combined with a half wave plate located between the telescope and AO188 constitutes a dual beam polarimeter. The PDI mode provides a powerful tool to find a proto-planetary/debris disk around the bright star since subtracting two images reduces the unpolarized speckle pattern of the bright star and only leaves the polarimetric signature of the disk. The SDI mode has two Wollaston prisms which split the light into four directions. The light in each direction is recorded through a separate filter located in front of the detector. The resulting image on the detector is then a collection of four images with $5^{\prime \prime} \times 5^{\prime \prime}$ each taken with four different filters at the same time. The SDI mode is effective to find a self-luminous gaseous planet bearing a spectroscopic feature such as methane absorption near the bright star as the subtraction of two or more images taken at different wavelengths enhances the spectroscopic feature of the planet while the speckle pattern of the bright star is subtracted out after appropriately scaling intensity and speckle pattern. Finally, in the ADI mode the rotation angle is controlled by an image rotator so as to keep the orientation of the pupil constant on the detector. As a result, the target star's PSF including the speckle pattern and the residual spider pattern remains fixed on the detector throughout the observation, while any planet in the field of view moves along an arc as the sky rotates. During data reduction, this sequence of images can be exploited to estimate and subtract the fixed stellar PSF while leaving the planet signals intact. Finally, the frames are de-rotated into a fixed position angle and co-added. An important advantage of the ADI mode is that the ADI technique works equally well on any kinds of self-luminous companions as it does not assume spectral feature as the SDI mode does.

This paper concentrates on reporting the performances of the instrument based on the data obtained in the laboratory and during the commissioning period. Imaging performance, detector performance, and coronagraphic performance are described in dedicated sections.

\section{DETECTOR PERFORMANCE}

\subsection{RAW IMAGE}

Figure 3 (left) shows a dark image as an example of the raw image. There are two significant features in the raw image due to the characteristics of the current readout system using a Sidecar ASIC and JADE2 card (Teledyne Scientific \& Imaging LLC.): 32 horizontal stripe patterns corresponding to 32 readout channels, and vertical banding patterns with a combination of some frequencies. Both patterns appear on the image in a random manner. In order to remove the horizontal stripe patterns, extra pixels called "reference pixels" are provided along the edges of the detector which have the same characteristics as rest of the pixels, but are not light sensitive. This "reference pixel subtraction" removes the horizontal stripe patterns almost completely. It also works for the vertical banding patterns, but some residuals persist as shown in Figure 3.

Another way to remove the patterns is to use a part of the image that has virtually no light signals. As the horizontal stripe patterns only differ in their offsets and the vertical banding patterns commonly exist in 32 horizontal stripes, extracting a common part of the patterns as a master pattern improves the pattern subtraction.
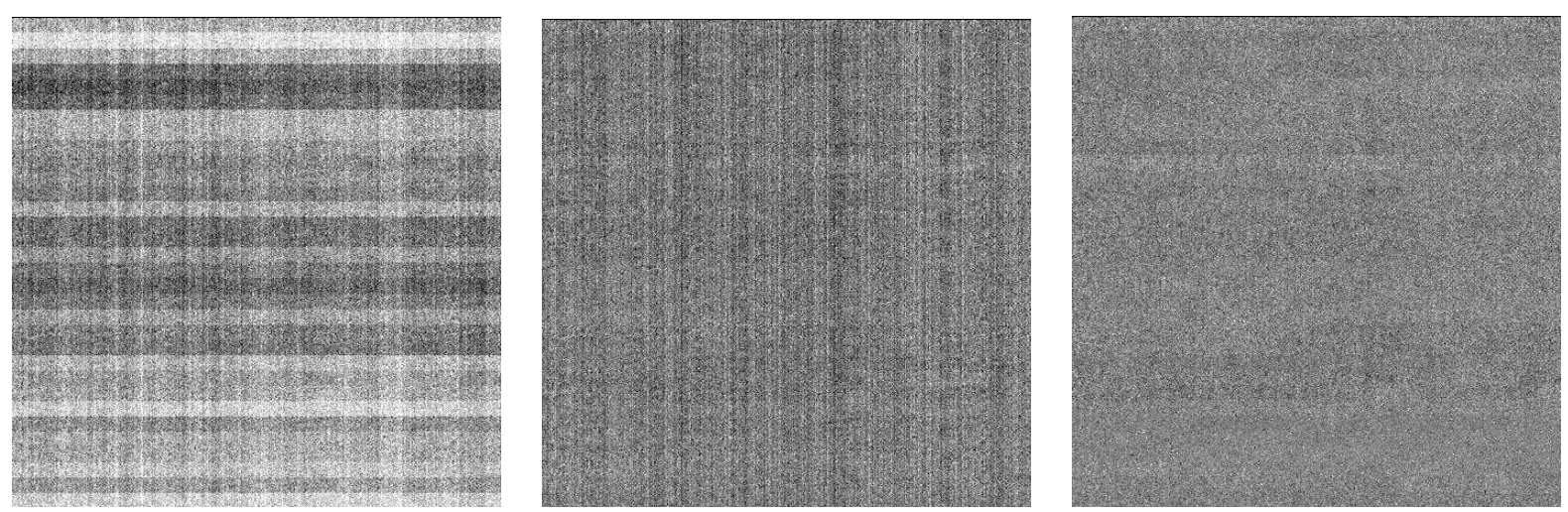

Figure 3. Left: Example of the raw image. Center: Image with the horizontal stripe patterns removed by "reference pixel subtraction". Right: Image with the horizontal and vertical patterns removed by "master pattern subtraction". 


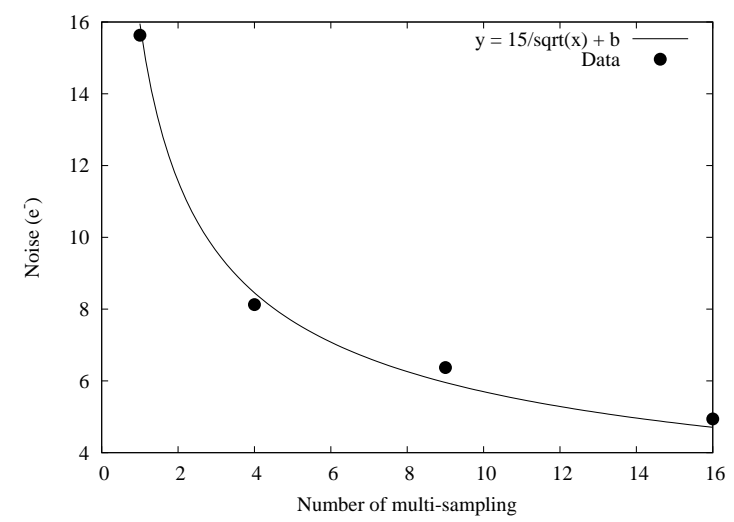

Figure 4. Readout noise with the multiple sampling method. A solid curve represents $1 / \sqrt{N}$ law.

A master horizontal stripe is created as a median image of the 32 horizontal stripes with a box filter after masking objects in the image. Subtracting the master stripe from each stripe after an appropriate offset removes the horizontal stripes and leaves only the vertical bandings in the image. A similar process creates a master vertical bandings from the 32 horizontal stripes without the box filter, and removes the pattern with much less residual as shown in Figure 3. We note that this "master pattern subtraction" method is only applicable under a certain condition where the detector has enough area with no light signals.

\subsection{GAIN}

Detector gain $g$ in $\mathrm{e}^{-} / \mathrm{ADU}$ is derived by a photon transfer curve which is expressed in a form,

$$
\sigma_{\text {total }}^{2}=\sigma_{\text {read }}^{2}+\frac{S}{g}
$$

where $\sigma_{\text {total }}$ is total noise, $\sigma_{\text {read }}$ readout noise, and $S$ signal, all in units of ADU. An aluminum plate at ambient temperature is used as a light source in the $K$-band. A least square fitting of the formula to the data yields the detector gain of $1.60 \mathrm{e}^{-} / \mathrm{ADU}$.

\subsection{READOUT NOISE}

Readout noise of the detector is measured using the dark image with the patterns removed following the "master pattern subtraction" method written in section 2.1. The readout noise of $15 \mathrm{e}^{-}$is obtained with a single correlated double sampling (CDS) method. Then we investigated how a multiple sampling method reduces the readout noise. Figure 4 shows the readout noise with different numbers of sampling. The readout noise follows a $1 / \sqrt{N}$ law at least to $N=16$ where we obtain readout noise of $5 \mathrm{e}^{-}$. It is noted that additional overhead of $2.8 N$ seconds are necessary for the multiple sampling method.

\subsection{DARK CURRENT}

Dark current is measured by taking dark images with different exposure times. A black-painted aluminum plate at $77 \mathrm{~K}$ was placed in front of the detector to prevent the light from reaching the detector. The detector is operated at $60 \mathrm{~K}$. The dark current measurement is not as straightforward as it is usually done because of the variable horizontal stripes described in section 2.1. The dark current is then derived from the total noise with different exposure times using the following formula,

$$
\sigma_{\text {total }}^{2}=\sigma_{\text {read }}^{2}+\frac{n_{\text {dark }} t}{g^{2}}
$$

where $\sigma_{\text {total }}$ is total noise in $\mathrm{ADU}, \sigma_{\text {read }}$ readout noise in $\mathrm{ADU}, n_{\text {dark }}$ dark current in $\mathrm{e}^{-} / \mathrm{s}, g$ detector gain derived in section 2.2, $t$ exposure time. A least square fitting of the formula provides the dark current of 0.046 $\mathrm{e}^{-} / \mathrm{s}$ which is sufficiently low for our purpose where exposure time barely exceeds a few minutes. 


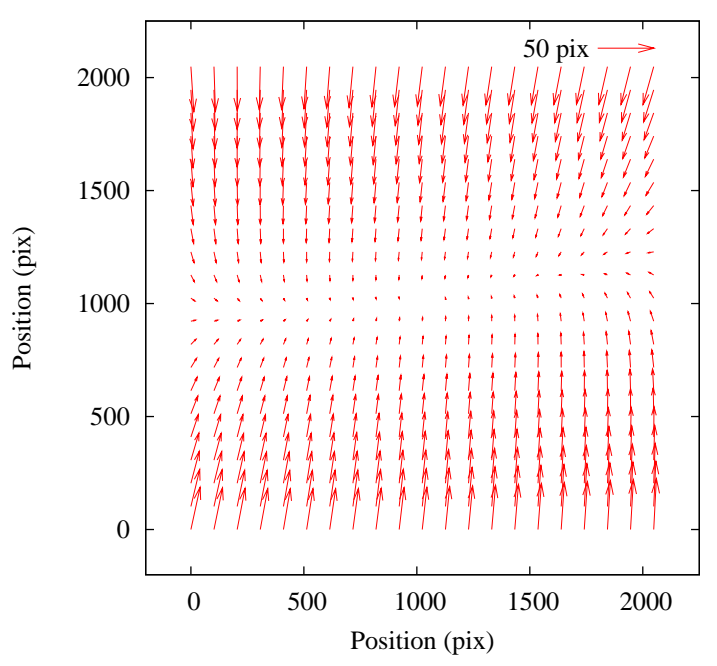

Figure 5. Distortion map of the DI mode image. Arrows show the amount of distortion for a grid pattern. The distortion is magnified 5 times for the illustrative purposes.

\section{IMAGING PERFORMANCE}

\subsection{STREHL RATIO}

Strehl ratio of AO188+HiCIAO is calculated by comparing peak-to-total flux ratio between observed and simulated PSFs. From a limited number of stars observed so far, we have obtained an average Strehl ratio of 0.4 and 0.67 in the $H$-band and $K$-band, respectively, with a guide star of $R \sim 5$ under natural seeing of $0.5^{\prime \prime}$ in the $H$-band. The Strehl ratio derived from the HiCIAO data is consistent with a result of other instrument for Subaru Telescope which works with AO188. FWHM of the PSF directly measured without assuming profile is $0.06^{\prime \prime}$ and $0.07^{\prime \prime}$ in the $H$-band and $K$-band, respectively. It is noted that the performance characterization efforts in the laboratory show that the Strehl ratio of HiCIAO itself is almost unity as expected from the optical design.

\subsection{DISTORTION}

We observed M15 to derive a distortion correction function in the DI mode. Coordinates of the stars extracted by SExtractor (Bertin \& Arnouts $1996^{5}$ ) are compared to those obtained by the ACS instrument on board the Hubble Space Telescope. We assume that the distortion of the ACS images is corrected at least at the level such that they can serve as reference images for HiCIAO. A fifth order polynomial for $\mathrm{x}$ and y coordinates are used to express the distortion correction function in a least square fitting process. The distortion pattern is characterized by a different pixel scale in $\mathrm{x}$ and $\mathrm{y}$ direction and non-linear effect corresponding to higher order terms. The difference in the pixel scale is $3 \%$, and this is consistent with the amount caused by a compensator which is a tilted wedged plate located in a converged beam. RMS of the residual of the object corrdinates from the best fit polynomial is 0.78 pix or 7 milli-arcsec in the entire $20^{\prime \prime} \times 20^{\prime \prime}$ field. The residual has a random pattern, and should be due to a measurement error of the centoid of HiCIAO PSFs.

\subsection{PIXEL SCALE}

Pixel scale is derived by measuring distance of a binary star with known angular distance in the pixel unit on the image. We observed a binary star (HIP 482) with 7.687 arcsec separation with the $H$-band in the DI mode. The distortion is corrected by the distortion correction function derived in section 3.2. Measurement at five different dithered positions gives the pixel scale of $9.406 \pm 0.004$ milli-arcsec/pix. The accuracy is again limited by the measurement error of the centroid of the HiCIAO PSFs. 


\subsection{THROUGHPUT}

Throughput is calculated using the following formula,

$$
\gamma=\frac{C_{\mathrm{obj}} g}{F_{\lambda, \mathrm{obj}} A_{\mathrm{tel}} \Delta \lambda}
$$

where $C_{\mathrm{obj}}$ is a count rate of an object in $\mathrm{ADU} / \mathrm{s}, g$ the detector gain in $\mathrm{e}^{-} / \mathrm{ADU}, F_{\lambda \text {,obj }}$ the photon flux of the object in photon $/ \mathrm{s} / \mathrm{cm}^{2}, A_{\text {tel }}$ the effective area of the telescope in $\mathrm{cm}^{2}$, and $\Delta \lambda$ the passband width of a filter in $\mu \mathrm{m}$. The throughput calculated by the formula above includes atmospheric transmission, telescope throughput, throughput of AO188, transmission of the instrument optics, and the detector QE. From a number of stars with known brightness observed at different zenith angles, the throughput at a zenith position is calculated as 0.09 , 0.11 , and 0.16 in the $J, H$, and $K$-band, respectively. The results agree with the numbers expected from the reflectivity of AR coatings, filter transmission, Lyot stop size, detector QE, and measured throughput of the telescope and AO188.

\subsection{BACKGROUND BRIGHTNESS}

We use background regions of the images taken with a long exposure time for the background brightness estimation. Zero points of the magnitude are used for conversion between the counts and the magnitude. The resulting background brightness is $15.2,13.2$, and $10.1 \mathrm{mag} / \operatorname{arcsec}^{2}$ (Vega) in the $J, H$, and $K$-band, respectively. The result in the $J$-band and the $H$-band is consistent with an average sky brightness of Mauna Kea, while the background in the $K$-band is brighter than the average because of thermal radiation from the optics at ambient temperature in $\mathrm{AO} 188$ and HiCIAO.

\subsection{LIMITING MAGNITUDE}

Limiting magnitude is calculated using the measured values described so far. The limiting magnitude with 1 hour exposure, $5 \sigma$ level, and $0.075^{\prime \prime}$ aperture is $24.05,23.69$, and $22.17 \mathrm{mag}$ (Vega) in the $J, H, K$-bands, respectively. The shallower limiting magnitudes compared to similar instruments on the other 8-m class telescopes originate from the undersized Lyot stop which cuts off $50 \%$ of the light collected by the telescope.

\subsection{GHOST}

Ghost image is investigated both in the laboratory and on the sky. In the laboratory using a light source with Strehl ratio of $\sim 1$, no ghost image brighter than $10^{-6}$ of the ghost source is found outside of $0.5^{\prime \prime}$ radius when the occulting mask is used in the DI mode. On the sky, there are two occasions where the ghost image has been found so far in the course of contrast analysis and ghost measurement analysis: one is an observation in the DI mode without the occulting mask, and the other is an observation in the SDI mode without the occulting mask. The ghost image in the DI mode is a pupil-like ghost with its contrast $10^{-5.6}$ of the ghost source per pixel and size 260 pix in radius. On the other hand, the ghost image in the SDI mode is a point-like ghost. It is generated by a double-bounce reflection of the light between the detector surface and the surface of the differential filter. The ghost can be found in all four channels of the SDI image. The location is either 0.31 or 0.67 from the ghost source depending on the channel, and the contrast is $10^{-4.3}$ of the ghost source.

\section{CONTRAST PERFORMANCE}

In this section, we demonstrate contrast performance in the different observing modes. Due to a limited observing time and variable atmospheric condition, it turns out difficult to make a representative contrast estimate for each observing mode. The results shown here should be considered as an example of performance that the instrument can achieve. Accumulation of the data in the future will enable more comprehensive performance evaluation for each observing mode as well as comparison between the observing modes. A summary of the observations is shown in Table 1.

The observation procedure of the coronagraphic observation is as follows: (1) slewing the telescope for target acquisition, (2) closing the AO loop and tuning the control parameters, (3) aligning the Lyot stop to the pupil in the pupil-viewing mode, (4) centering the object on the occulting mask, and (5) starting science exposures. 
Table 1. Summary of the observations for the contrast evaluation.

\begin{tabular}{lccccccc}
\hline \hline Object & Observing mode & Filter & Occulting mask & Exposure time & $R$ & $H$ & $K$ \\
\hline GJ 564 & DI & $H$ & $0.6^{\prime \prime}$ & $160 \mathrm{~s}$ & 5.5 & 4.7 & 4.5 \\
GJ 564 & DI & $K$ & $0.6^{\prime \prime}$ & $720 \mathrm{~s}$ & 5.5 & 4.7 & 4.5 \\
HIP 104214 & SDI & $\mathrm{CH}_{4}$ & $0.3^{\prime \prime}$ & $500 \mathrm{~s}$ & 4.5 & 2.5 & 2.2 \\
HD 282411 & PDI & $H$ & $0.3^{\prime \prime}$ & $? ? \mathrm{~s}$ & - & 4.6 & 4.1 \\
GJ 564 & ADI+DI & $H$ & $0.6^{\prime \prime}$ & $360 \mathrm{~s}$ & 5.5 & 4.7 & 4.5 \\
GJ 564 & ADI+DI & $K$ & $0.6^{\prime \prime}$ & $780 \mathrm{~s}$ & 5.5 & 4.7 & 4.5 \\
HR 8799 & ADI+DI & $H$ & No & $525 \mathrm{~s}$ & - & 5.3 & 5.2 \\
\hline
\end{tabular}

The PSF image was visually monitored, and any tip/tilt error was corrected with a pixel accuracy during the exposures.

We follow standard data reduction processes such as flat fielding, bad/hot pixel correction, cosmic ray rejection, and distortion correction after the pattern subtraction process described in section 2.1. A different recipe is then employed to remove the speckle pattern for each observing mode as each observing mode has a different feature in the speckle pattern. The distortion is corrected using the distortion correction function described in 3.2 for the DI and ADI mode, while the distortion correction function derived using a grid pinhole pattern placed on the focal plane of AO188 (i.e., where occulting mask is placed) is used for the PDI and SDI mode.

For consistent contrast measurement, we use the same method to create contrast curves. (1) First, the image with the speckle pattern removed goes through a smoothing filter with an aperture whose size is roughly same as a PSF core of the filter used (e.g., $5 \times 5$ pixels in the $H$-band). (2) Then the image is divided into octant regions centered on the object position. (3) A standard deviation of the pixel counts is calculated within a circular ring with 5 pixel width in each octant region along radial distance from the object. (4) The standard deviations are multiplied by 5 to account for a detection with $\mathrm{S} / \mathrm{N}=5$. (5) Finally, the $5 \sigma$ deviations are either converted to surface brightness relative to the total magnitude of the central star (in case of the PDI mode) or divided by the peak count of the object PSF obtained without the occulting mask (in other observing modes).

\subsection{MODE}

We have observed GJ 564 with the $H$ and $K$-band in the DI mode. The target is known to host a binary brown dwarf at $2.6^{\prime \prime}$ separation. The total exposure time is $160 \mathrm{~s}$ and $720 \mathrm{~s}$ in the $H$ and $K$-band, respectively. The occulting mask with $0.6^{\prime \prime}$ diameter was used for better observation efficiency. As the simplest way to subtract the PSF with the speckle pattern, we rotate the image by 180 degree and subtract it from the original one. The resulting image and the contrast curves are shown in Figure 6 and Figure 7. The contrast is $10^{-3.54}$ at $r=0.4^{\prime \prime}$, $10^{-4.78}$ at $r=1.0^{\prime \prime}, 10^{-5.43}$ at $r=1.5^{\prime \prime}, 10^{-5.90}$ at $r=2.0^{\prime \prime}$, and $10^{-6.08}$ at $r=2.5^{\prime \prime}$ in the $H$-band, and $10^{-3.94}$ at $r=0.4^{\prime \prime}, 10^{-4.74}$ at $r=1.0^{\prime \prime}, 10^{-5.37}$ at $r=1.5^{\prime \prime}, 10^{-5.48}$ at $r=2.0^{\prime \prime}$, and $10^{-5.48}$ at $r=2.5^{\prime \prime}$ in the $K$-band. The contrast is limited by the speckle noise in $r<2.0^{\prime \prime}$ and by the readout noise in the outer area. This method is obviously too simple to achieve high contrast especially in the speckle-dominated inner region. However, it could be still useful in certain cases, for example, when the main interest lies in an outer region and the target cannot provide enough field rotation for the ADI mode.

\subsection{PDI MODE}

We have observed HD 282411, which is a nearby non-polarized star with $H=4.6$, in the $H$-band for the contrast measurement in the PDI mode. The stable and excellent seeing condition enables us to use the occulting mask with $0.3^{\prime \prime}$ diameter. The data with the total exposure time of ?? $\mathrm{s}$ is obtained at each of four position angles $(0$, $22.5,45$, and 67.5 degree) of the half wave plate. Two $10^{\prime \prime} \times 20^{\prime \prime}$ images (we call "channel images") extracted from a PDI frame are processed in the standard way. The distortion correction and image registration between the two channel images uses the pinhole grid data. Then two sets of Stokes $Q$ and $U$ images, i.e., $+Q,-Q$, 

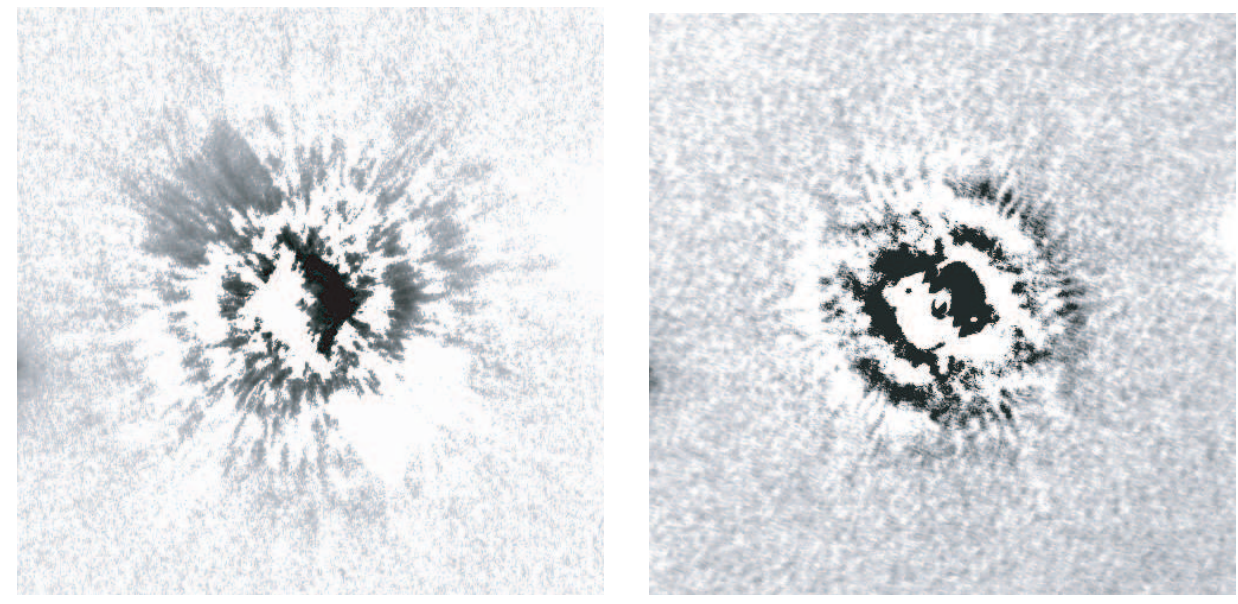

Figure 6. Images of fully reduced data for GJ 564 in the DI mode ( $H$-band $(l e f t)$ and $K$-band $(l e f t))$. Image size is $5^{\prime \prime} \times 5^{\prime \prime}$.
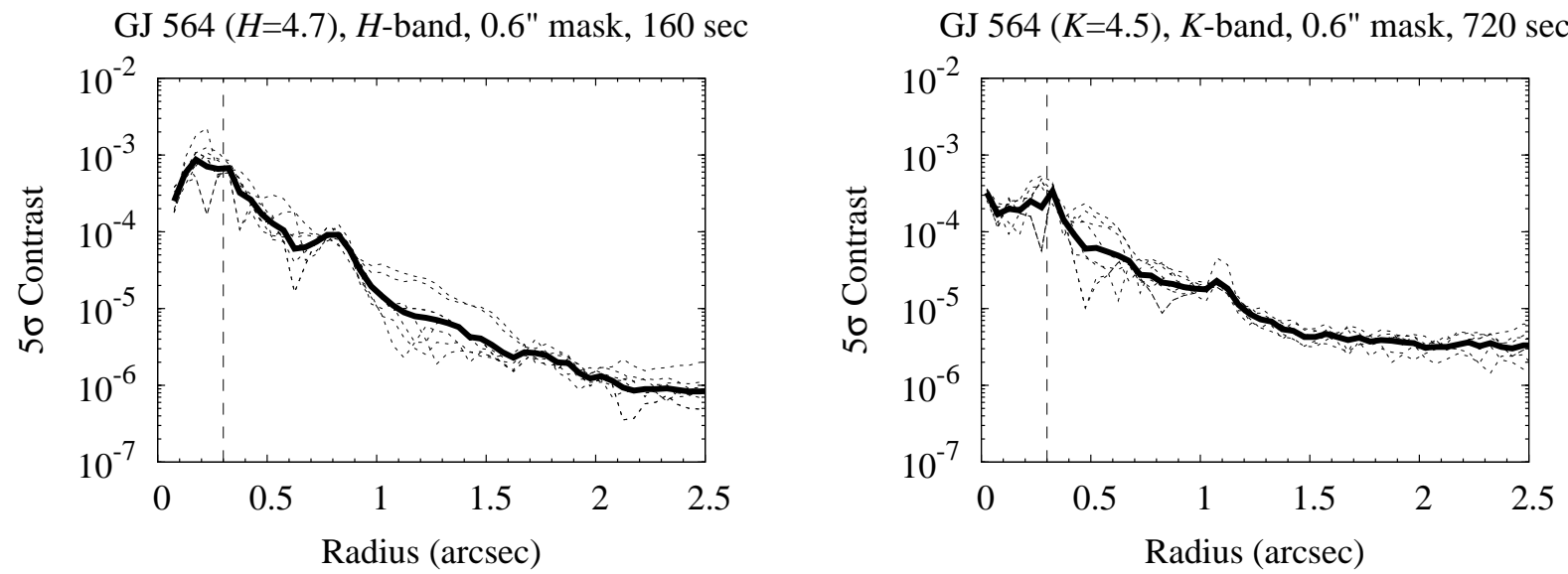

Figure 7. Contrast curve for GJ 564 in the DI mode ( $H$-band (left) and $K$-band (right)). Thin dotted curves represent the result in each octant region, while the thick solid curve is their average. A vertical dashed line shows the size of the occulting mask.

$+U$, and $-U$, are created by subtracting the two channel images for $0,45,22.5$ and 67.5 degree, respectively. Finally, $P I$ image is produced as $P I=\sqrt{Q^{2}+U^{2}}$ where $Q=((+Q)-(-Q)) / 2$ and $U=((+U)-(-U)) / 2$. The resulting $P I$ image and relative surface brightness to the total magnitude of the central star corresponding to 5 $\sigma$ detection limit are shown in Figure 8. The relative surface brightness is $8.7 \mathrm{mag} / \operatorname{arcsec}^{2}$ at $r=0.2^{\prime \prime}, 9.0$ at $R=0.4^{\prime \prime}, 11.4$ at $r=1.0^{\prime \prime}, 12.2$ at $r=1.5^{\prime \prime}, 12.5$ at $r=2.0^{\prime \prime}$, and 12.5 at $r=2.5^{\prime \prime}$. The readout noise becomes a dominant noise source at $r>2.0^{\prime \prime}$. It is noted that there is no systematic pattern even near the edge of the occulting mask in the PI image, indicating that polarization can be measured as close to as $0.15^{\prime \prime}$ from the star.

\subsection{SDI MODE}

We have observed HIP 104214 for the contrast performance evaluation in the SDI mode. The occulting mask with 0.3 " diameter was used because the atmospheric condition and the seeing in the night were stable and excellent. With a set of methane narrowband filters centered on $\lambda=1.575,1.600,1.625,1.644 \mu \mathrm{m}$, we obtained the SDI images with total exposure time of $500 \mathrm{~s}$.

After the standard image process described above, four $5^{\prime \prime} \times 5^{\prime \prime}$ images in a SDI frame are extracted as an independent image (we call four images "channel images"). Distortion correction is applied separately to each 

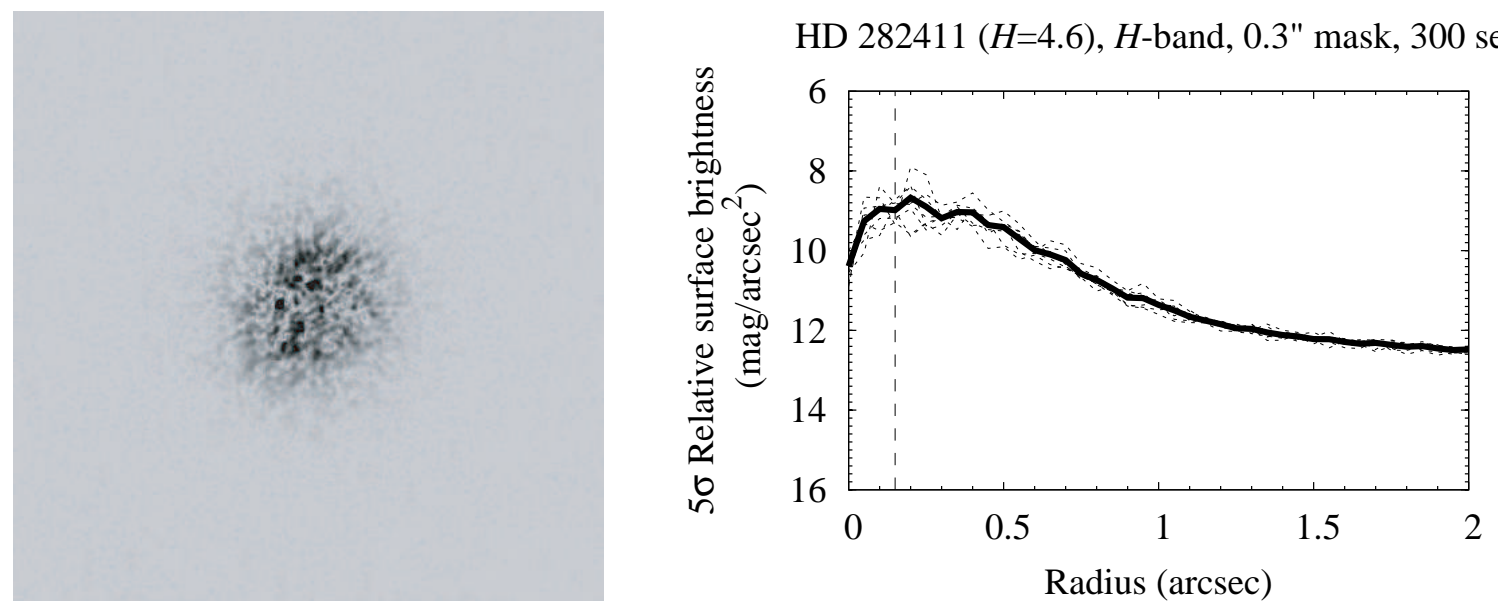

Figure 8. Contrast performance in the PDI mode. Left: PI image of HD 282411. Image size is $5^{\prime \prime} \times 5^{\prime \prime}$. Right: Surface brightness curve corresponding to $5 \sigma$ detection limit. Definition of the lines is same as Figure 7 .

channel image as the distortion pattern is different among the channels. Speckle subtraction in the SDI mode requires special consideration because (1) the speckle pattern around the primary star is different from channel to channel due to a scaling effect by the wavelength, and (2) scaling in intensity is also necessary as each image is taken with a different filter. A linear transformation (shift, rotation, and scale) is considered in matching the speckle patterns between the channel images. The best parameters in the linear transformation are determined from a range of parameter spaces such that sum of the squared subtraction residuals is minimized.

Figure 9 shows the SDI image with speckle pattern subtracted and the contrast curves for HIP 104214. Subtraction of the two channel images with $\lambda=1.600$ and $\lambda=1.575$ is shown as an example, however, we confirm that the results with different wavelength combinations agree within a factor of 2 . The contrast is $10^{-3.52}$ at $r=0.2^{\prime \prime}, 10^{-4.04}$ at $r=0.4^{\prime \prime}, 10^{-4.90}$ at $r=1.0^{\prime \prime}, 10^{-5.00}$ at $r=1.5^{\prime \prime}, 10^{-5.05}$ at $r=2.0^{\prime \prime}$. Although the sensitivity of the SDI mode is quite low due to the narrowband filters, it is still the most powerful observing mode to find self-luminous gaseous planets with the spectroscopic feature, and more importantly, the SDI mode
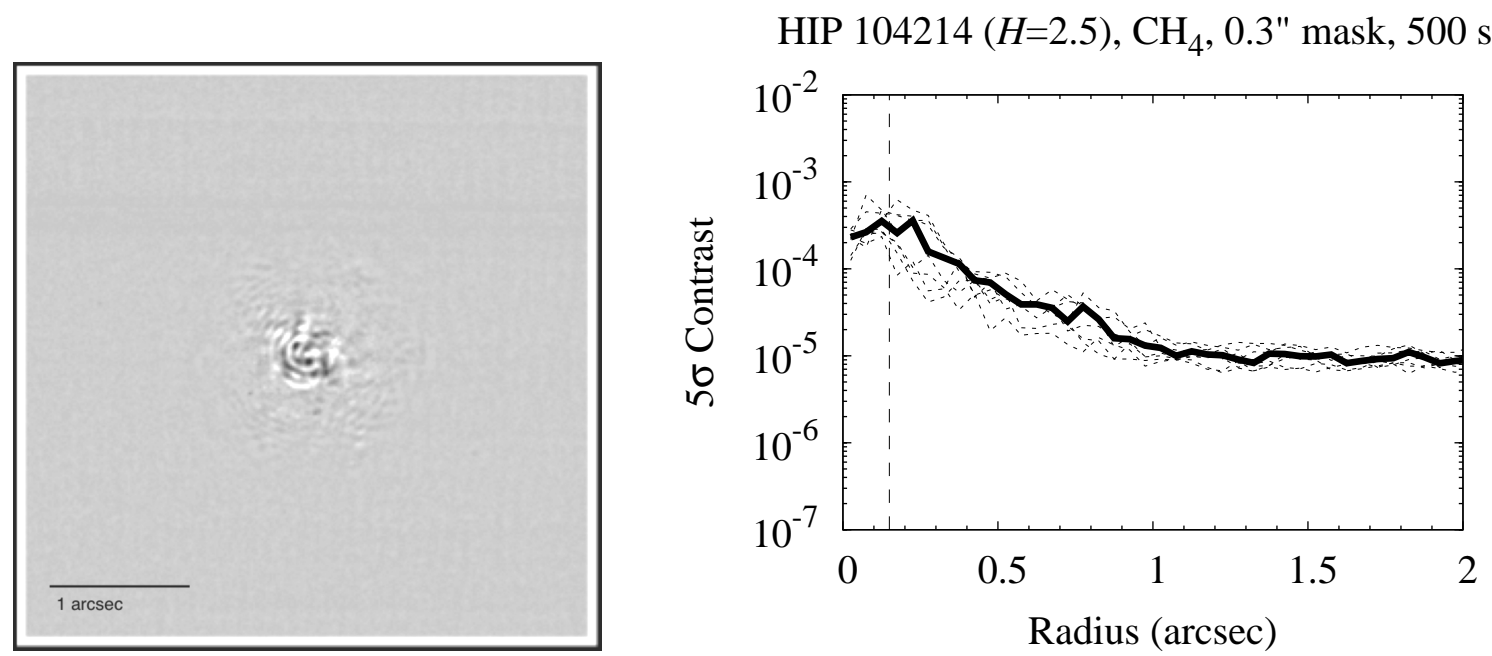

Figure 9. Contrast performance in the SDI mode. The result of $\lambda=1.600-\lambda=1.575$ is shown as an example. Left: High contrast image of HIP 104214. Image size is $4^{\prime \prime} \times 4^{\prime \prime}$. Right: Contrast curve. Definition of the lines is same as Figure 7 . 

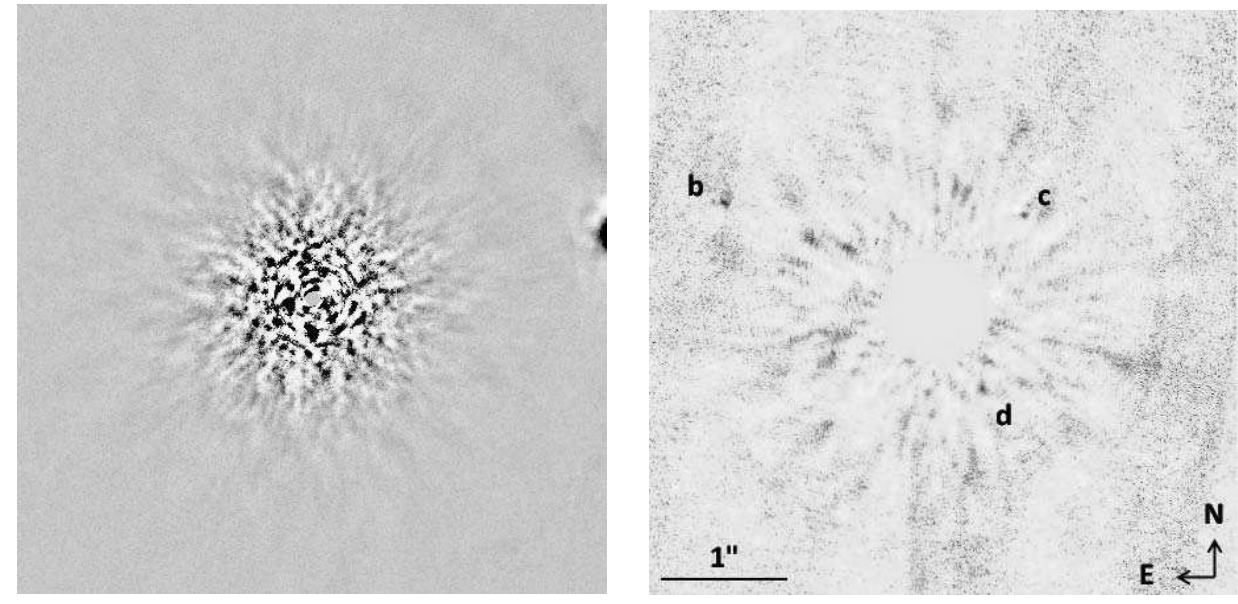

Figure 10. Left: Fully reduced $K$-band image of GJ 564 in the ADI mode. Image size is $5^{\prime \prime} \times 5^{\prime \prime}$. Right: Signal-to-noise map of the $H$-band image of HR 8799 in the ADI mode. Image size is $5^{\prime \prime} \times 5^{\prime \prime}$. Three objects labeled a,b,c are planets discovered by Marois et al. (2008). ${ }^{7}$
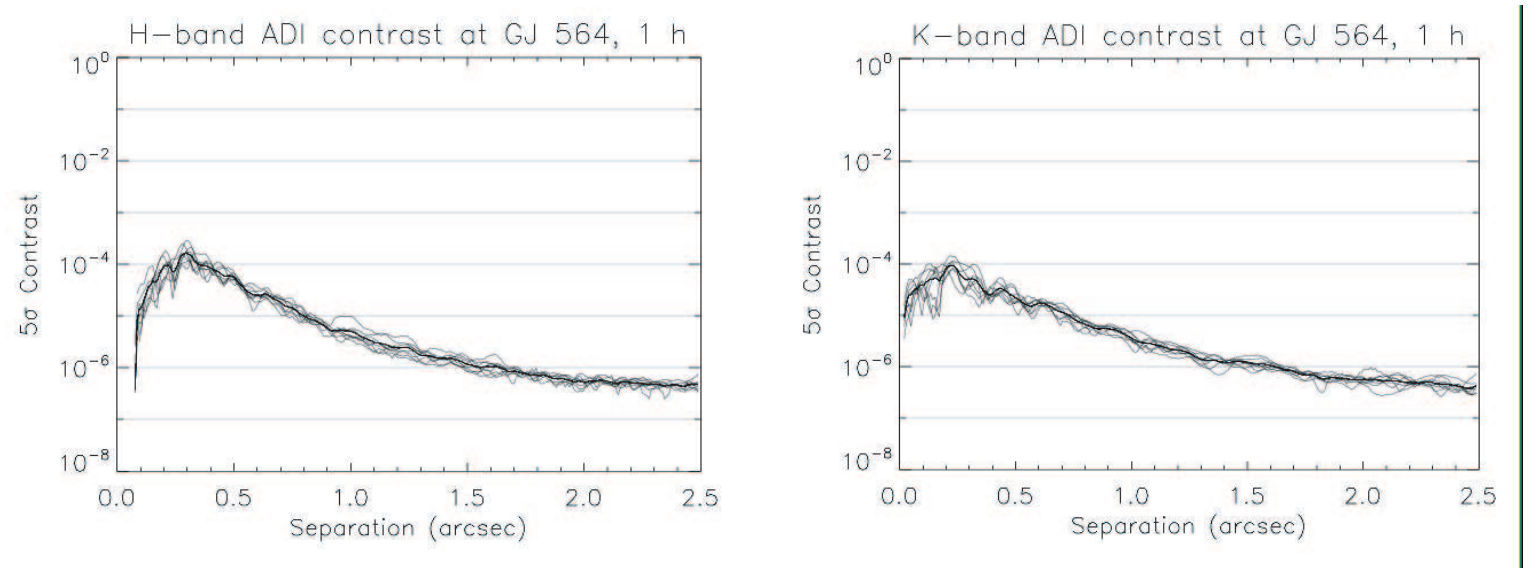

Figure 11. Contrast curve for GJ 564 in the ADI mode ( $H$-band (left) and $K$-band (right)). Definition of the lines is same as Figure 7.

can explore the most inner region than the other observing modes due to less chromatic effect on the speckle pattern.

\subsection{ADI MODE}

We have observed GJ 564 and HR 8799 for the contrast evaluation in the ADI mode. GJ 564 was observed in the $H$ and $K$-band with $0.6^{\prime \prime}$ occulting mask, while HR 8799 was observed in the $H$-band without the occulting mask. The exposure time is $360 \mathrm{~s}$ and $720 \mathrm{~s}$ in the $H$ and $K$-band, respectively for GJ 564, and $525 \mathrm{~s}$ for HR 8799. The data are reduced using a custom set of IDL routines that implement the "LOCI" algorithm (Locally Optimized Combination of Images, Lafrenière et al. 2007 $)$. As a very conservative form of high-pass spatial filtering, a radial profile is subtracted from each frame. The LOCI code then divides each frame into annuli and segments, and a localized background was subtracted for each frame. Each segment background is computed from all other frames in the sequence by applying an optimization process that minimizes the image residuals. Finally, the resulting frames are de-rotated to position angle zero and co-added to produce the high-contrast output image.

Figure 10 shows a fully reduced $K$-band image of GJ 564 . The contrast performance curves for the GJ 564 in the $H$ and $K$-band are presented in Figure 11. We note that the contrast curve is divided by the "partial 
Table 2. Performance summary of HiCIAO

\begin{tabular}{ll}
\hline \hline Detector gain & $1.60 e^{-} / \mathrm{ADU}$ \\
Readout noise & $15 e^{-}$ \\
Dark current & $0.046 \mathrm{e}^{-} / \mathrm{s}$ \\
Strehl ratio & $0.4(H), 0.7(K)$ \\
Distortion & See Figure 5 \\
Distortion correction & 7 milli-arcsec $(\mathrm{rms})$ in $20^{\prime \prime} \times 20^{\prime \prime}$ \\
Pixel scale & $9.406 \pm 0.004 \mathrm{milli}-\operatorname{arcsec} / \mathrm{pix}$ \\
Throughput & $0.09(J), 0.11(H), 0.16(K)$ \\
Background brightness & $15.4(J), 13.2(H), 10.1(H) \mathrm{mag} / \mathrm{arcsec}^{2}$ \\
Limiting magnitude & $24.05(J), 23.69(H), 22.17(K) \mathrm{mag}$ \\
\hline
\end{tabular}

subtraction factor", a separation-dependent parameter between 0 and 1 describing the percentage of planet flux that survives the LOCI subtraction, which is calculated by inserting test companions into the raw data and processing it through the LOCI pipeline. The measured contrast is $10^{-}$at $r=0.4^{\prime \prime}, 10^{-}$at $r=1.0^{\prime \prime}, 10^{-}$at $r=1.5^{\prime \prime}, 10^{-}$at $r=2.0^{\prime \prime}$, and $10^{-}$at $r=2.5^{\prime \prime}$ in the $H$-band, and $10^{-}$at $r=0.4^{\prime \prime}, 10^{-}$at $r=1.0^{\prime \prime}, 10^{-}$ at $r=1.5^{\prime \prime}, 10^{-}$at $r=2.0^{\prime \prime}$, and $10^{-}$at $r=2.5^{\prime \prime}$ in the $K$-band. Figure 10 also shows a detection of three planets around HR 8799 discovered by Marois et al. (2008). ${ }^{7}$ This is a good demonstration of AO188+HiCIAO's capability as only 9 minutes exposure made a clear detection of the planet companions.

Although the target position on the sky comes into a consideration to take a full advantage of field rotation, the ADI mode is the most powerful observing mode in searching the planet over the wide field coverage without assuming planet property. Another benefit is that the ADI mode can be combined with other observing modes to possibly enhance the contrast performance.

\section{SUMMARY}

We describe imaging performance, detector performance, and coronagraph performance of the HiCIAO instrument based on the data obtained in the laboratory and during the commissioning observations. HiCIAO is the high contrast instrument designed to work with the 188 element adaptive optics for the Subaru Telescope. HiCIAO utilizes the adaptive optics, coronagraph, and differential imaging techniques to achieve the high contrast imaging observation near the bright stars for the extrasolar planet search and detailed disk sciences.

The imaging performances are mostly consistent with the design and satisfactory for our purpose. The highlights of the imaging performances are summarized as follows. (1) The Strehl ratio of 0.4 and 0.7 in the $H$ and $K$-band is obtained with $R \sim 5$ star under $0.5^{\prime \prime}$ seeing condition in the $H$-band. (2) The distortion pattern is correctable to 7 milli-arcsec accuracy which is limited by measurement accuracy of the PSF centroid. (3) The background brightness is consistent with the average sky brightness at Mauna Kea in the $J$ and $H$-band, but the $K$-band (10.1 mag/ $\left.\operatorname{arcsec}^{2}\right)$ is brighter due to the warm optics in AO188 and HiCIAO.

The detector performance is characterized by $1.60 \mathrm{e}^{-} / \mathrm{ADU}$ detector gain, $15 \mathrm{e}^{-}$readout noise, $0.046 \mathrm{e}^{-} / \mathrm{s}$ dark current, and some patterns on the raw image. The readout noise becomes as low as $5 \mathrm{e}^{-}$using the multiple readout method. The pattern appearing on the raw image can be removed almost completely using two methods depending on the situation.

Finally, we present the contrast performance for each observing mode. Due to a limited number of data obtained during the commissioning period, the results shown here should be considered as an example of the performance that the instrument can achieve. We demonstrate that (1) the PDI mode and SDI mode can explore the inner region of the object PSF to $0.15^{\prime \prime}$ and (2) the ADI mode is effective enough to detect the planets around HR 8799 with exposure time less than 10 minutes. Future observations will provide fairer representation of the contrast performance under different conditions such as atmospheric conditions, target brightness, occulting mask size. 


\section{ACKNOWLEDGMENTS}

We are grateful to the HiCIAO Science working group member and reviewers in the early phase of the instrument development, especially, M. Fukagawa, M. Hayashi, J. Kasdin , J. Krist, H. Kataza, M. Kuchner, M. Liu, T. S. Pyo, B. Sato, A. Sivaramakrishnan, E. Turner, M. Ueno, T. Yamamoto for their contributions in the CDR and PDR. We are grateful to the previous coronagraph (CIAO) development and operation teams, especially, N. Kaifu, M. Ishii, N. Ebizuka, S. S. Hayashi, K. Murakawa, Y. Itoh, and N. Takato. We also thank the Subaru telescope team for their telescope/instrument supports. This instrument development was supported by the MEXT Grant-in-Aid for Scientific Research on Priority Areas, "Development of Extra-Solar Planetary Science". The strategic observations with this instrument are supported by the MEXT Grant-in-Aid for Specially Promoted Research. M.T. acknowledges support from The Mitsubishi Foundation.

\section{REFERENCES}

[1] Hayano, Y., Takami, H., Oya, S., Hattori, M., Saito, Y., Watanabe, M., Guyon, O., Minowa, Y., Egner, S. E., Ito, M., Garrel, V., Colley, S., Golota, T. I., and Iye, M., "Commissioning status of Subaru laser guide star adaptive optics system," Proc. SPIE 7736 (2010).

[2] Hodapp, K. W., Tamura, M., Suzuki, R., Jacobson, S., Stahlberger, V., Yamada, H., Takami, H., Guyon, O., and Abe, L., "Design of the HiCIAO instrument for the Subaru Telescope," Proc. SPIE 6269, 123 (2006).

[3] Hodapp, K. W., Suzuki, R., Tamura, M., Abe, L., Suto, H., Kandori, R., Morino, J., Nishimura, T., Takami, H., Guyon, O., Jacobson, S., Stahlberger, V., Yamada, H., Shelton, R., Hashimoto, J., Tavrov, A., Nishikawa, J., Ukita, N., Izumiura, H., Hayashi, M., Nakajima, T., Yamada, T., and Usuda, T., "HiCIAO: the Subaru Telescope's new high-contrast coronographic imager for adaptive optics," Proc. SPIE 7014, 42 (2008).

[4] Tamura, M., Hodapp, K. W., Takami, H., Abe, L., Suto, H., Guyon, O., Jacobson, S., Kandori, R., Morino, J., Murakami, N., Stahlberger, V., Suzuki, R., Tavrov, A., Yamada, H., Nishikawa, J., Ukita, N., Hashimoto, J., Izumiura, H., Hayashi, M., Nakajima, T., and Nishimura, T., "Concept and science of HiCIAO: high contrast instrument for the Subaru next generation adaptive optics," Proc. SPIE 6269, 28 (2006).

[5] Bertin, E. and Arnouts, S., "SExtractor: Software for source extraction," A\&AS 117, 393 (1996).

[6] Lafrenière, D., Marois, C., Doyon, R., Nadeau, D., and Artigau, E., "A New Algorithm for Point-Spread Function Subtraction in High-Contrast Imaging: A Demonstration with Angular Differential Imaging," ApJ 660, 770 (2007).

[7] Marois, C., Macintosh, B., Barman, T., Zuckerman, B., Song, I., Patience, J., Lafrenière, D., and Doyon, R., "Direct Imaging of Multiple Planets Orbiting the Star HR 8799," Science 322, 1348 (2008). 